\title{
Data Mining Tools Used in Deep Brain Stimulation - Analysis Results
}

\author{
Oana Geman \\ Faculty of Electrical Engineering and Computer Science, \\ "Stefan cel Mare" University, \\ Suceava, Romania \\ geman@eed.usv.ro
}

\begin{abstract}
Parkinson's disease is associated with motor symptoms, including tremor. The DBS (Deep Brain Stimulation) involves electrode implantation into sub-cortical structures for long-term stimulation at frequencies greater than $100 \mathrm{~Hz}$. We performed linear and nonlinear analysis of the tremor signals to determine a set of parameters and rules for recognizing the behavior of the investigated patient and to characterize the typical responses for several forms of DBS. We found patterns for homogeneous group for data reduction. We used Data Mining and Knowledge discovery techniques to reduce the number of data. To support such predictions, we develop a model of the tremor, to perform tests determining the DBS reducing the tremor or inducing tolerance and lesion if the stimulation is chronic.
\end{abstract}

Keywords: Parkinson's disease, Deep Brain Stimulation, Data Mining.

\section{Parkinson's Disease, Tremor and Deep Brain Stimulation}

Parkinson's disease (PD) is a serious neurological disorder with a large spectrum of symptoms (rest tremor, bradykinesia, muscular rigidity and postural instability). The neurons do not produce dopamine anymore or produce very low level of this chemical mediator, necessary on movement coordination. Parkinson's disease seems to occur in about 100 - 250 cases on 100000 individuals. In Europe were reported about 1.2 million Parkinson patients [1]. The missing of a good clinical test, combined with the patient's reticence to attend a physician, make the diagnostic to be established very often too late. The accuracy of the diagnosis of PD varies from 73 to 92 percent depending on the clinical criteria used.

Parkinsonian tremor is a rhythmic, involuntary muscular contraction characterized by oscillation of a part of the body. Initial symptoms include resting tremor beginning distally in one arm at a $4-6 \mathrm{~Hz}$ frequency. Deep Brain Stimulation (DBS) is an electric therapy approved by FDA (Food and Drug Administration) for the treatment of Parkinson's disease (PD) in 2002 and used now to treat the motor symptoms like essential tremor. It consists of a regular high frequency stimulation of specific subcortical sites involved in the movement-related neural patterns [1].

The exact neurobiological mechanism by which DBS exerts modulator effects on brain tissue are not yet full understood. It is unknown which part of the neuronal 
structure (cell body, axon) is primarily modulated by DBS. Since the causes are confused, the treatment does not provide cure but slows down the illness evolution and attenuate the invalidating symptoms - tremor.

\section{Methods}

The current study was motivated by the wish to develop a better understanding of potential, mechanisms of the effects of Deep Brain Stimulation on Parkinsonian tremor by studying the tremor dynamics that occur during the on/off of high frequency DBS in subjects with Parkinson's disease.

Currently there are insufficient data in the literature to include data and specific knowledge of nonlinear dynamics, which would eventually lead to the improvement of Parkinson's disease database, to get an accurate a medical diagnosis, with rare exceptions [2], [3], [4]. The field of nonlinear dynamics introduced idea that a random behavior of tremor time series might have been generated by a low-dimensional chaotic deterministic dynamical system. Parkinsonian tremor exhibits a nonlinear oscillation that is not strictly periodic. Titcombe et al. [2] applied various methods from linear and nonlinear time series analysis to tremor time series.

First, we made a linear, nonlinear and statistical analysis of the tremor signals (available on the internet at [5]) to determine a set of parameters and rules for recognizing the behavior of the investigated patient and to characterize the typical responses for several forms of DBS. Second, we found representatives for homogeneous groups in order to perform data reduction. Then we found "clusters" and describe their unknown properties. We used k-means and k-medoids clustering.

Therefore, it is desirable to predict, before we pursuit with this type of operation, if the DBS will give good results in a specific case. To make such predictions, we need a model of the tremor, on which to perform tests to determine if the DBS procedure will reduce the tremor. An accurate, general model of a system can allow the investigation of a wide variety of inputs and behaviors in sillico, without the time or resources required to perform the same investigations in vivo.

\section{Clinical Databases Structure}

Parkinsonian tremor time series are available on internet [5]. These data were recorded using a low-intensity velocity laser in subjects with Parkinson's disease receiving chronic, high frequency DBS at a sampling rate of $100 \mathrm{~Hz}$. Tremor signals were recorded to the subject's index finger in a resting position continuously throughout switching the DBS on and off.

We analyzed tremor signals from 16 subjects with Parkinson disease, ages between 37 and 71 years, 11 men and 5 women. The recordings of this database are of rest tremor velocity in the index finger of 16 subjects with Parkinson's disease (PD) who receive chronic high frequency electrical Deep Brain Stimulation (DBS) either uni- or bi-laterally within one of three targets: Vim = the ventro-intermediate nucleus of the thalamus, $\mathrm{GPi}=$ the internal Globus pallidus, $\mathrm{STN}=$ the subthalamic nucleus. 


\section{Data Analysis Results}

\subsection{Linear and Nonlinear Time Series Analysis of Parkinsonian Tremor}

Linear analysis of the signal is mainly using Fourier analysis and reporting made comparison of the amplitude frequency bands. Based on the Fourier spectrum in the range of $0-25 \mathrm{~Hz}$ and amplitude-time representation, a number of parameters are used to characterize the tremor signal [6], [7]. Many reports suggest that Parkinsonian tremor is typically in the range of 4-6 Hz, and the essential tremor is in the range of 4$12 \mathrm{~Hz}$.

A very first phase on non-linear analysis is to draw the phase diagram. In the signal period, the phase diagram is a closed curve. If the signal is chaotic, the diagram is a closed curve called strange attractor. The positive Lyapunov exponent is the main chaotic dynamic indicator. In case at least one Lyapunov exponent is bigger that 0 , the system is chaotic. Using CDA software solution to analyze the tremor signals from our database, we found the Lyapunov exponent varies from 0.02 to 0.28 , depending on the signal type (Fig.1 shows the Lyapunov exponent (a), fractal dimension (b), and correlation dimension (c)).

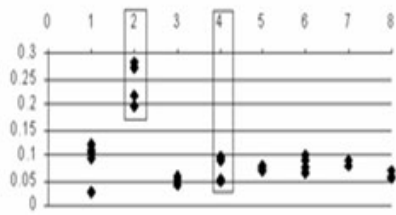

a

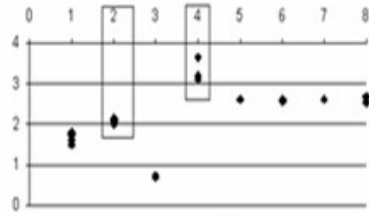

b

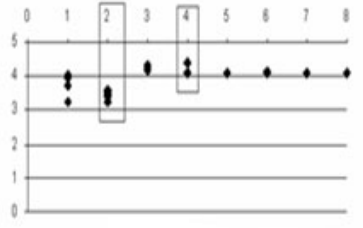

C

Fig. 1. The values of Lyapunov exponent (a), fractal dimension (b), and correlation dimension (c) (class 2 and class 4 are marked because results will be analyzed)

We have 8 preset classes of tremor: class 1 - DBS on/medication on; class 2 - DBS on/medication off; class 3 - DBS off/medication on; class 4 - DBS off/medication off (Parkinsonian tremor); class 5...8 - DBS off/medication off (the signals recorded after 15, 30, 45 and, respectively, 60 minutes after ceasing the DBS action).

Analyzing the data, we observed that the fractal dimension allows us to recognize with good accuracy the high-amplitude tremor classes. Using the other parameters/rules, the classification becomes more accurate.

Based on the information (for example the values of Lyapunov exponent - Fig. 1 (a)) we may conclude: using the values of the Lyapunov exponent, we may distinguish class 2 (DBS on/medication off) from all the other classes. In this case the values of Lyapunov exponent are between 0.19 and 0.28 (1).

Using the values of the fractal dimension, we observe that in case of class 4 (DBS off/medication off - simple Parkinsonian tremor) there are important differences relative to other classes (for class 4, values between 3.2 and 3.8) (2). Using the correlation dimension, we notice that for class 2 (DBS on/medication off), its values are between 3.3 and 3.7 and for class 4 (DBS off /medication off) the correlation dimension can reach a value of 4.5 (this value does not exist for any other class) (3). 
In conclusion, for the patients with Parkinson's disease, using the correlation dimension and calculation of Lyapunov exponents, we found evidence that the dynamics are generated by a nonlinear deterministic and chaotic process. We argue that nonlinear dynamic parameters of Parkinsonian tremor have certain peculiarities and can be used in knowledge-discovery. This data and new knowledge will be integrated in a Knowledge-based System aimed to identify each class of tremor, to worn on atypical responses at DBS and to recommend the stimulation targets (thalamus nuclei).

\subsection{Data Mining Tools}

Data Mining has been defined as the automatic analysis of a large and complex data sets in order to discover meaningful patterns. Recent advances in Data Mining research has led to developement of efficient methods for finding patterns and important knowledge in large volumes of data, efficient method of classification, clustering, frequent pattern analysis, sequential, structured [8]. This raises the acute need to link the two areas of Arificial Intelligence: Bioinformatics and Data mining for efective analysis of biomedical data, with aim of discovering new knowledge and data in the medical field.

Cluster analysis tools based on k-means, k-medoids, and several other methods have also been buit into many statistical analysis software systems. Given a data set of $\mathrm{n}$ objects, and $\mathrm{k}=$ the number of clusters to form, a partitioning algorithm organizes the objects into k partitions, where each partition represents a cluster.

We adopt two of a few popular heuristic methods, such as:

1. the k-means algorithm, where each cluster is represented by the mean value of the objects in the cluster;

2. the k-medoids algorithm, where each cluster is represented by one of the objects located near the center of the cluster.

Because it is easier to illustrate, but also because, classes 2 and 4 are the most significant (class 2 - signals acquired from patients tremor with DBS without medication, class 4 - patients without medication and without DBS, Parkinsonian Tremor), we will continue this k-means algorithm and k-medoids algorithm only for these classes.

Using STATISTICA software version 9 for classes 2 and 4 we obtained the following results:

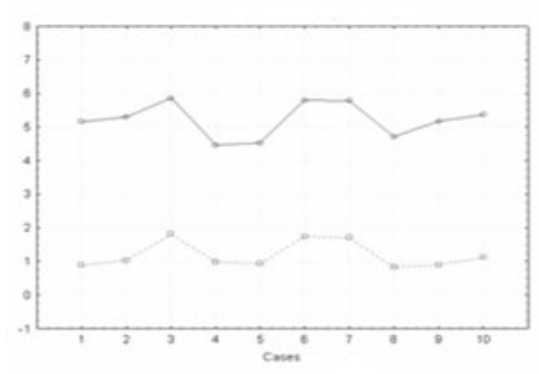

a

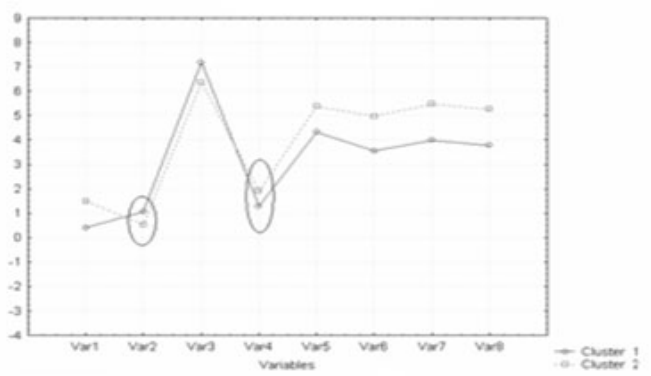

b

Fig. 2. K-means Clustering Algorithm (a) and K-medoids Clustering Algorithm (b) for class 2 and 4 (cluster 1 (a)/var2 (b) is class 2 - DBS on/medication off and cluster 2 (a)/var4 (b) is class 4 - DBS off, medication off) 
We have shown that applied k-means clustering algorithm and k-medoids clustering algorithm solve the well known clustering problem and DBS treatments results identify. Being an invasive method it is important to predict patient's response to DBS and therefore the next step is to model the Parkinsonian tremor.

\subsection{A Nodel of the Parkinsonian Tremor}

Modeling of biological systems is difficult due to nonlinearities, time dependence and internal interactions common in physiological and neural systems. To get a true understanding of the behavioral of a complex biological systems technique of nonlinear dynamical systems modeling can be used. Modeling complex biological systems is very difficult, but the simplest method for predicting output from input is the linear regression. We proposed the usage of a nonlinear iterated function in modeling simple biological processes. Controlling the parameters of the nonlinear function, we control the dynamic of the generated process [9].

We notice that between the original Parkinson tremor and the model there are visible similarities up to the time scale. The model based on adaptive systems searching the matching Lyapunov exponent, is a preliminary model, which can be further improved [9]. Developing an accurate nonlinear dynamical model of a patient's PD symptoms as a function of DBS could both increase the effectiveness of DBS and reduce the clinical time needed to program the implanted stimulator [10], [11], [12]. If the model were able to accurately predict the level of symptoms as a result of past stimulation, the model could be used to test all possible parameter settings very quickly in simulation to identify the globally optimal stimulus parameters.

\section{Conclusions and Future Works}

As it has been demonstrated, classes 2 and 4 are easy to identify, which reinforces the following conclusions: only ordinary statistical parameters are not enough for identifying the tremor classes (1); the nonlinear dynamic parameters are not sufficient in distinguishing between different tremor classes (2); a more careful analysis of elements from point 1 and 2 helps to a more easy class identification (3); refinement of current indication for DBS procedure is possible, regarding the risks and benefits of STN versus Gpi DBS for PD (4).

To determine if the DBS procedure will reduce the tremor, we need a model of the tremor, on which to perform tests. The results obtained are encouraging and we will continue the research on this issue, by modeling other known nonlinear processes. We also demonstrated that, by iterating a linear function, we could model a Parkinsonian tremor, and we developed an adaptive system to build this model.

Acknowledgments. This paper was supported by the project "Progress and development through post-doctoral research and innovation in engineering and applied sciences - PRiDE - Contract no. POSDRU/89/1.5/S/57083", project cofunded from European Social Fund through Sectorial Operational Program Human Resources 2007-2013. 


\section{References}

1. National Parkinson Foundation, http: / /www. parkinson. org

2. Titcombe, M.S., Glass, 1., Guehl, D., Beuter, A.: Dynamics of Parkinsonian tremor during Deep Brain Stimulation. Chaos 11(4), 201-216 (2001)

3. Teodorescu, H.N., Kandel, A.: Nonlinear Analysis of Tremor and Applications. JJME Japanese Journal of Medical Electronics and Biological Engineering 13(5), 11-19 (2004)

4. Teodorescu, H.N., Chelaru, M., Kandel, A., Tofan, I., Irimia, M.: Fuzzy methods in tremor assessment, prediction, and rehabilitation. Artificial Intelligence in Medicine 21(1-3), 107130 (2001)

5. PhysioNet, http://www. physionet.org/database

6. Schlapfer, T.E., Bewernick, B.H.: Deep brain stimulation for psychiatric disorders - state of the art. In: Advances and Technical Standards in Neurosurgery, vol. 34, pp. 115-211. Springer, Heidelberg (2002)

7. Rocon, E., Belda-Lois, J.M., Sancez-Lacuesta, J.J., Pons, J.L.: Pathological tremor management: Modelling, compensatory technology and evaluation. Technology and Disability 16, 3-18 (2004) ISSN 1055-4181/04

8. Danubianu, M.: Advanced Databases. In: Modern Paradigms in Computer Science and Applied Mathematics, p. 30. AVM, Munchen (2011) ISBN: 978-3-86306-757-1

9. Geman (Voroneanu), Teodorescu, H.N., Zamfir, C.: Nonlinear Analysis and Selection of Relevant Parameters in Assessing the Treatment Results of reducing Tremor, using DBS procedure. In: Proceedings of International Joint Conference on Neural Networks, Paper 1435 (2004)

10. Tuomo, E.: Deep Brain Stimulation of the Subthalamic Nucleus in Parkinson Disease, a Clinical Study, faculty of Medicine, University of Oulu, Finland (2006)

11. Van Battum, E.Y.: The Role of Astrocytes in Deep Brain Stimulation, Master thesis, Master Neuroscience and Cognition (2010)

12. Elias, J.W., Lozano, A.M.: Deep Brain Stimulation: The Spectrum of Application, Neurosurg. Focus, 29(2), American Association of Neurological Surgeon (2010) 